\title{
Multi-messenger searches with the ANTARES and KM3NeT neutrino telescopes
}

\author{
Marta Colomer Molla*, on behalf of the ANTARES Collaboration \\ APC, Univ Paris Diderot, CNRS/IN2P3, CEA/Irfu, Obs de Paris, Sorbonne Paris Cité, France \\ IFIC-Instituto de Física Corpuscular (CSIC - Universitat de València), Valencia, Spain \\ E-mail: mcolomereapc.in2p3.fr
}

\begin{abstract}
The year 2017 brought important breakthroughs to the astro-particle community, the first detection of an electromagnetic counterpart of the gravitational wave signal from a binary neutron star merger and the potential correlation between high-energy neutrinos observed by the IceCube telescope and the flaring blazar TXS 0506+056. These observations have opened a new way to probe extreme phenomena in the sky. Combination of these messengers is now possible and fundamental information can be obtained with these new probes.
\end{abstract}

The ANTARES neutrino telescope has been operating for more than ten years in the Mediterranean sea with the purpose of searching for high energy cosmic neutrinos. During the last few years the multi-messenger approach has delivered intriguing new results for Cherenkov neutrino detectors with promising potential for future astrophysical searches. Thus, the ANTARES Collaboration is actively participating to the follow up of alerts sent by different experiments covering the full electromagnetic spectrum, IceCube and the gravitational-wave interferometers. This allows probe the potential neutrino emission from various sources, including fast radio bursts, gamma-ray bursts, binary mergers and blazars. ANTARES also sends alerts that lead to an electromagnetic follow-up of interesting neutrino events. The latest results will be discussed in the following.

The New Era of Multi-Messenger Astrophysics - Asterics2019

25 - 29 March, 2019

Groningen, The Netherlands

\footnotetext{
*Speaker.
} 


\section{Introduction}

The ANTARES neutrino telescope is located at $40 \mathrm{~km}$ offshore of Toulon, in France, at a depth of almost $2500 \mathrm{~m}$ in the Mediterranean sea. The deployment was completed in 2008 and it has been taking data for more than 10 years already. It consists of 12 lines with 25 storeys per line and 3 photo-multipliers per storey. Neutrino telescopes continuously monitor at least half of the sky, so they are well suited to look for transient sources. The short duration of the fluxes from these transient and variable sources allow to reduce the background and increase our discovery potential.

\section{The Multi-messenger program of ANTARES}

The Multi-Messenger (MM) program of ANTARES is based on two different approaches. The first approach consists in triggering an alert to the observer partners as soon as a neutrino event is detected. This is the aim of the so-called TAToO program of ANTARES. This part will not be covered here but the interested reader can refer to [1]. The second one consists in doing the followup of the alerts received from other experiments. For interesting events, we look for neutrinos in coincidence in space and time. The ANTARES collaboration is active on the neutrino follow-up of the IceCube neutrino detections, the alerts from the LIGO-Virgo detectors and the EM detections of fast radio bursts, blazars or Gamma-Ray Bursts (GRBs) among others. These time dependent searches are done both in real time and offline.

\section{Search for neutrinos in correlation with Fast Radio Bursts}

Fast Radio Bursts (FRBs) are one of the most intriguing and energetic phenomena in the Universe. While their origin and mechanism is still unknown, they are believed to be potential sources of high-energy neutrinos.

A search for neutrinos correlated in space and time with fast radio bursts detected from 2013 to 2017 has been performed using ANTARES data [2]. No significant correlation is found and upper limits are put on the neutrino fluence assuming different energy spectrums. The ANTARES $90 \%$ confidence level (CL) upper limits (ULs) on the neutrino fluence are presented in Fig. 1 as a function of the neutrino energy for 3 different energy spectrums, for the FRBs in the field of view.

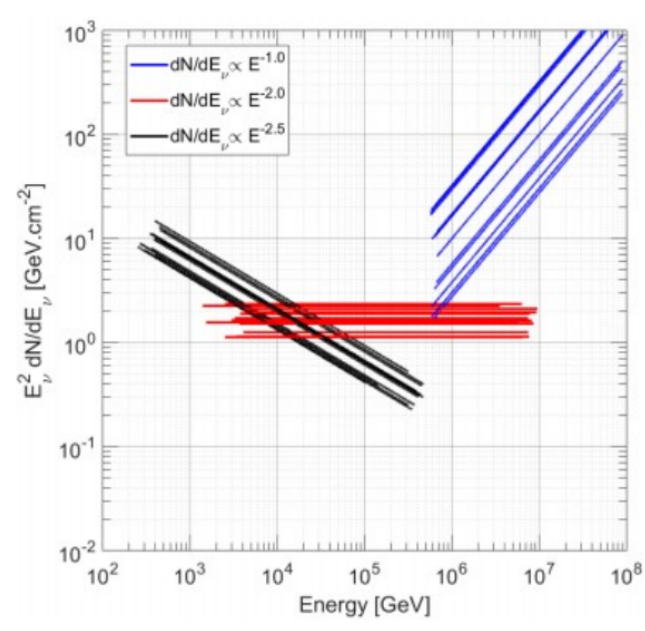

Figure 1: The 90\% CL ANTARES ULs on the neutrino fluence, for an $\mathrm{E}^{-1}$ (black), $\mathrm{E}^{-2}$ (red) and $\mathrm{E}^{-2.5}$ (blue) energy spectrums, for the 12 FRBs analyzed.

\section{Search for a counterpart to IceCube events with ANTARES}

\subsection{Time-correlation with IceCube events}

A search for time correlations with IceCube (IC) events from the HESE and the high-energy $v_{\mu}$ tracks samples as been performed with ANTARES to test the transient origin of IC events. 
No significant correlation has been found over a timescale of less than $3 \mathrm{~h}$, with the largest excess being a 89\% post trial p-value, as reported in [4]. From this null result, the spectral index of the neutrino spectrum can be constrained. On Fig.2, we show the 90\% UL on the expected number of signal events in IC originating from a transient point-like source emitting in a time-window smaller than 0.1 days, as a function of the spectral index $(\gamma)$ for the highest energy event of each sample (a HESE event in blue and a $v_{m u}$ track in red. These IC events are only consistent with a transient origin for neutrino spectra harder than -2.3 and -2.4 , correspondingly.

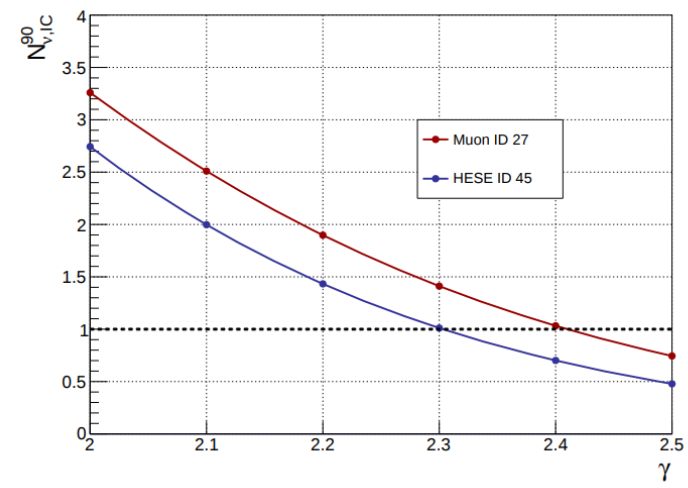

Figure 2: The $90 \%$ ULs on the expected number of IC events originating from a transient source emitting in a time window $\leq 0.1$ days as a function of the spectral index $\gamma$ for the most energetic IC event of each sample. The dotted line indicates 1 event detected by IC.

\subsection{Search for neutrinos from TXS $0506+506$}

A dedicated search for neutrinos from TXS 0506+506 has been performed and detailed in [5] triggered by the potential observation by IceCube of a high-energy neutrino (IC170922a) in coincidence with the flaring blazar. For this special event, three different analysis were performed. An online search yielded no up-going neutrino candidate within $3^{\circ}$ around the direction of the IC event within $\pm 1 \mathrm{~h}$ centered on the event time. With the standard point-source method (see [6]), 13 tracks and 1 shower neutrino candidates were found within $5^{\circ}$ from TXS. The results obtained using 10 years of ANTARES data are a number of fitted signal events of 1.03 and a pre-trial p-value of $3.4 \%$.

Table 1: Upper limits on the neutrino flux for the time dependent analysis.

\begin{tabular}{|c|c|c|}
\hline Spectrum & $\phi_{100 T e V}^{90 \%}\left[\mathbf{1 0}^{-18} \mathbf{G e V}^{-1} \mathbf{c m}^{-2} \mathbf{s}^{-1}\right]$ & $\mathbf{5 \% - 9 5 \%}$ energy range \\
\hline $\mathrm{E}^{-2}$ & 4.6 & $2.0-3.2 \mathrm{TeV}$ \\
\hline $\mathrm{E}^{-2.1}$ & 4.4 & $1.3-1.6 \mathrm{TeV}$ \\
\hline $\mathrm{E}^{-2.2}$ & 4.2 & $1.0-1.0 \mathrm{TeV}$ \\
\hline
\end{tabular}

A time dependent search has also been applied for this source. A variable neutrino source compatible with the excess of events found by IC between 2014 and 2015 at the location of TXS 0506+506 was searched in the archival ANTARES data. The search is done with two different burst shapes modelling the neutrino light-curve, a Gaussian and a Box flare, as defined in [7]. Although 13 events are found in the data within $2^{\circ}$ from the source, none of them lie within either of the two flaring periods considered. The upper limits on the neutrino flux normalization are shown in Table 1 for the energy range where $90 \%$ of the events are contained. 


\section{Search for neutrinos in correlation with graviational-wave events}

In 2015, the first gravitational-wave (GW) signal coming from the merging of two black holes was detected. Since then, $11 \mathrm{GW}$ events have been cataloged [8]. ANTARES actively followed all of the events for which an alert was sent and is currently participating to the follow-up of the events detected during the third observational run of Advance-LIGO/Virgo. The good angular resolution of ANTARES $\left(0.4^{\circ}\right.$ at $\mathrm{E}_{v}>10 \mathrm{TeV}$ for tracks) could help, in the case of an associated neutrino discovery, to reduce the GW location error box and thus allow for a rapid pointing to look for an electromagnetic counterpart to GW events.

\subsection{Neutrino follow-up of binary Black-Hole events: the case of GW170104}

Neutrino emission coincident with Binary Black-Hole $(\mathrm{BBH})$ events can take place if an hadronic environment and/or a strong magnetic field allow for particle acceleration on this sites $[10,11]$. A dedicated offline analysis was done for the first GW detection (GW150914) as well as for GW151226 during O1 [12].

The first detection from run $\mathrm{O} 2$, GW170104, also led to an offline search by ANTARES with an increase of sensitivity due to the optimised data quality cuts used in this analysis. For the first time, events above the ANTARES horizon were included in the analysis. No event in correlation was found and full sky upper limits are derived as explained in [9]. Results are shown in Fig.3 assuming an $\mathrm{E}^{-2}$ neutrino spectrum.

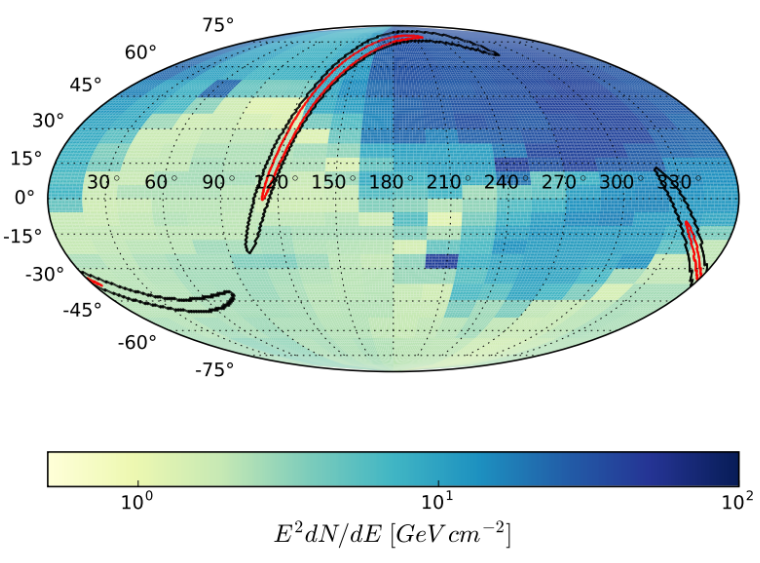

Figure 3: All-sky upper limit on the neutrino spectral fluence $\left(v_{\mu}+\overline{v_{\mu}}\right)$ from GW170104 as a function of source direction assuming $\mathrm{dN} / \mathrm{dE} \propto \mathrm{E}^{-2}$ neutrino spectrum. The red and black lines show the GW skymap contours at $50 \%$ and $90 \%$ credible levels, respectively. The skymap is defined in equatorial coordinates.

\subsection{Follow-up of the binary neutron star merger: GW170817}

High-energy neutrino emission from binary neutron star (BNS) mergers might be expected if an ejection process with an hadronic component is related to the merger event or if there is cosmic-ray acceleration related to a magnetar.

The first BNS (GW170817), was followed, 2 seconds later, by a short GRB and a kilonova was later observed over the full electromagnetic spectrum [13, 14]. It was an special event that brought light on the physics riding this astrophysical phenomena, only $40 \mathrm{Mpc}$ away.

The event was jointly followed-up by ANTARES, IC and Auger detectors. Two analyses were performed, one over a short time window of $\pm 500 \mathrm{~s}$ and another over an extended window of 14 days. It is the first ANTARES all-flavor GW analysis. No event in coincidence with the GW event was found and upper limits on the neutrino spectral fluence are set over the energy range covered.

Full results can be found in [16]. In Fig. 4, the upper limits obtained by each of the 3 experiments are compared to predictions from theoretical models. Relying on [15], an on-axis jet neutrino emission would have been observed by IceCube for the optimistic scenario. 

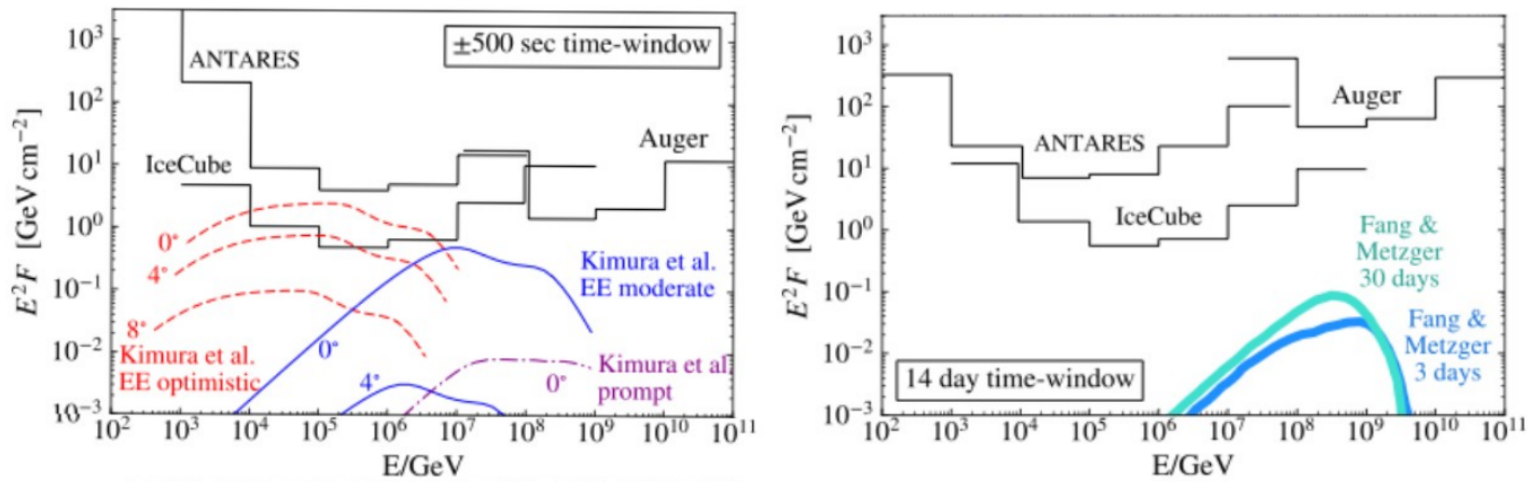

Figure 4: Upper limit on the neutrino spectral fluence $\left(v_{\mu}+\overline{v_{\mu}}\right)$ from GW170817 as a function of the neutrino energy assuming an $\mathrm{E}^{-2}$ neutrino spectrum, for the short time window search $( \pm 500 \mathrm{~s}$, left $)$ and for the extended search over 14 days (right). They are given for the IceCube, ANTARES and Auger detectors.

\section{Conclusions and outlook}

Neutrino telescopes can increase their discovery potential and sensitivity by combining their measurements with the ones from other messengers and by requiring time and space correlation in the search. The ANTARES detector has been actively participating on the exiting MM programs and has brought competitive results t constrain the transient neutrino sky. The KM3NeT detectors, being deployed in the Mediterranean sea, will represent a step forward in terms of technology and expected performances. As a result, the expectations for MM searches are promising, aiming at detecting neutrino transient sources and proving them as high-energy cosmic rays accelerators.

\section{References}

[1] M.Ageron et al., Astroparticle Physics, Volume 35, p.530-536., 2012

[2] Albert et al. ,MNRAS 482, 2019

[3] Biehl, D., Heinze, J., \& Winter, MNRAS, 476, p. 1191-1197, 2018

[4] Albert et al., arXiv:1902.09462

[5] Albert et al., Astrophys.J. 863 (2018) no.2, L30

[6] Albert, A. et al., Phys.Rev. D96 (2017) no.8, 082001

[7] IceCube Collaboration, Science, Vol. 361, Issue 6398, pp. 147-151, 2018

[8] Ligo-Virgo Collaboration, GWTC-1, arXiv:1811.12907 (2018)

[9] Albert et al., Eur. Phys. J. C (2017) 77: 911

[10] Kumiko Kotera and Joseph Silk, Astrophys.J., 823(2):L29, 2016

[11] R.Perna, D.Lazzati, B. Giacomazzo, Astrophys.J. 821 (2016) no.1, L18

[12] ANTARES, IceCube and LIGO-Virgo Collaborations, Phys.Rev. D93 (2016) no.12, 122010 \& Phys.Rev. D96 (2017) no.2, 022005

[13] LIGO-Virgo, Fermi-GBM and INTEGRAL Collaborations, Astrophys.J. 848 (2017) no.2, L13, 2017

[14] I.Arcavi, G.Hosseinzadeh et al., Nature, Volume 551, pages 64-66

[15] Kimura et al., Astrophys.J. 848 (2017) no.1, L4

[16] ANTARES and IC and Pierre Auger and LIGO-Virgo Collaborations, Astrophys.J. 850 (2017) no.2 